\title{
Role of the Band Gap for the Interaction Energy of Coadsorbed Fragments
}

Castelli, Ivano Eligio; Man, Isabela-Costinela; Soriga, Stefan-Gabriel; Parvulescu, Vasile; Halck, Niels Bendtsen; Rossmeisl, Jan

Published in:

The Journal of Physical Chemistry Part C

Link to article, DOI:

10.1021/acs.jpcc.7b04974

Publication date:

2017

Document Version

Peer reviewed version

Link back to DTU Orbit

Citation (APA):

Castelli, I. E., Man, I-C., Soriga, S-G., Parvulescu, V., Halck, N. B., \& Rossmeisl, J. (2017). Role of the Band Gap for the Interaction Energy of Coadsorbed Fragments. The Journal of Physical Chemistry Part C, 121(34), 18608-18614. https://doi.org/10.1021/acs.jpcc.7b04974

\section{General rights}

Copyright and moral rights for the publications made accessible in the public portal are retained by the authors and/or other copyright owners and it is a condition of accessing publications that users recognise and abide by the legal requirements associated with these rights.

- Users may download and print one copy of any publication from the public portal for the purpose of private study or research.

- You may not further distribute the material or use it for any profit-making activity or commercial gain

- You may freely distribute the URL identifying the publication in the public portal 


\title{
The Role of the Band Gap for the Interaction Energy of Coadsorbed Fragments
}

\author{
Ivano E. Castelli, ${ }^{\dagger}$ Isabela-Costinela Man, ${ }^{*} \ddagger$ Stefan-Gabriel Soriga ${ }^{\S}$ Vasile \\ Parvulescu, ${ }^{\ddagger}$ Niels Bendtsen Halck,,$\|$ and Jan Rossmeis|*,†
}

Nano-Science Center, Department of Chemistry, University of Copenhagen, DK-2100, Copenhagen Ø, Denmark, University of Bucharest, Faculty of Chemistry, Department of Organic Chemistry, Biochemistry and Catalysis, 4-12 Regina Elisabeta Av., S3, RO-030018

Bucharest, Romania, Romanian Academy, "C.D. Nenitzescu" Center of Organic

Chemistry, 202B Spl. Independentei, RO-060023 Bucharest, Romania, University

Politehnica of Bucharest, Centre for Technology Transfer in the Process Industries, 1, Gh. Polizu Street, Building A, Room A056, RO-011061 Bucharest, Romania, and Department of Energy Conversion and Storage, Technical University of Denmark, Building 309, DK-2800, Kgs. Lyngby, Denmark

E-mail: isabela.man@g.unibuc.ro, isabela.traistaru@ccodn.ro(I.C.M.); jan.rossmeisl@chem.ku.dk(J.R.)

\footnotetext{
${ }^{*}$ To whom correspondence should be addressed

${ }^{\dagger}$ Nano-Science Center, Department of Chemistry, University of Copenhagen, DK-2100, Copenhagen $\varnothing$, Denmark

${ }^{\ddagger}$ University of Bucharest, Faculty of Chemistry, Department of Organic Chemistry, Biochemistry and Catalysis, 4-12 Regina Elisabeta Av., S3, RO-030018 Bucharest, Romania

"Romanian Academy, "C.D. Nenitzescu" Center of Organic Chemistry, 202B Spl. Independentei, RO060023 Bucharest, Romania

$\S$ University Politehnica of Bucharest, Centre for Technology Transfer in the Process Industries, 1, Gh. Polizu Street, Building A, Room A056, RO-011061 Bucharest, Romania

"Department of Energy Conversion and Storage, Technical University of Denmark, Building 309, DK2800, Kgs. Lyngby, Denmark
} 


\begin{abstract}
Understandings of the interaction between adsorbants and metal surfaces have led to descriptors for bindings and catalysis which have a major impact on the design of metal catalysts. On semiconductor oxides these understandings still lack. We show an important element in understanding binding on semiconductors. We propose here a correlation between the cooperative interaction energy, i.e. the energy difference between the adsorption energies of coadsorbed electron donor-acceptor pair and isolated fragments and the band gap of the clean oxide surface. We demonstrate this effect for a number of oxides and donor-acceptor pairs and explain it with the shift in the Fermi level before and after the adsorption. The conclusion is that the adsorption of acceptordonor pairs is considerably more favorable compared to unpaired fragments and this energy difference is approximately equal to the value of the band gap. The implications of this understanding in relation with the improvement and discovery of novel catalysts on the band gap oxides are also discussed.
\end{abstract}

\title{
Introduction
}

Research in theoretical heterogeneous catalysis has rapidly grown in the last 20 years. ${ }^{1-7}$ Scaling relationships, which mainly describe correlations between adsorption properties of reactants and intermediates across different materials, are important tools that facilitate rational design of catalysts on metals by predicting trends in activity and selectivity across material surfaces for a wide range of application. ${ }^{8-17}$ The main class of materials on which scaling relationships for heterogeneous catalysis have been applied are pure transition metal surfaces. ${ }^{18-20}$ Extensions of this concept have also been applied to other catalytic surfaces, like alloys ${ }^{21-23}$ and oxides. ${ }^{15,24}$ In general, the role of the electronic structure in determining the adsorption properties, which for metals is to a large extent understood, is still matter of debate in case of semiconducting and insulating surfaces (since insulators are large band gap semiconductors and to avoid redundancies in the text, from now on, we indicate band 
gap materials with the general term semiconductors.

Fragments coadsorbed on oxides are found to behave in a very different way compared to what is seen on metals. If in the case of metal surfaces, the computational study of catalytic process was simplified by calculating the binding energy of each molecular fragment alone on the surface, without registering significant changes when fragments are coadsorbed, in the case of oxides it was found to fail dramatically. In fact, a Lewis-acid (A, whose electron charge increases during a reaction) and a Lewis-base ( $\mathrm{B}$, that loses charge) stabilize each other through the semiconductor oxide surface (attractive interaction or cooperative adsorption) when they are coadsorbed. ${ }^{25-29}$ In other words, the energy of coadsorbing A and B at large, but finite, distance is much more negative, i.e. energetically favorable, than the case where there is an infinite distance between the two fragments corresponding to the situation in which each fragment is adsorbed alone (cooperative adsorption). This interaction is small when the fragments are coadsorbed on a conductor. ${ }^{30}$

This effect has been pointed out for few systems, for example, $\mathrm{NO}_{x}$ adsorption on alkalineearth oxide surfaces, ${ }^{25,31-36}$ halogen and halogen hydrides on $\mathrm{CeO}_{2}(111)$ and $\mathrm{La}_{2} \mathrm{O}_{3}(001),{ }^{28,29}$ methane activation on $\mathrm{La}_{2} \mathrm{O}_{3}(001)$ and $\mathrm{PdO},{ }^{37}$ and $\mathrm{H}-\mathrm{OH}$ pair on $\mathrm{CeO}_{2}(111), \mathrm{BaO}(100)$, $\mathrm{TiO}_{2}(110)$, and $\mathrm{PdO}(101) .{ }^{38}$ Forward steps are also done in understanding the mechanism of the cooperative adsorption by investigating, for example, charge transfers, electrostatic interactions, and ionic relaxations. ${ }^{38}$

Here, we report an understanding of the adsorption on oxide semiconducting surfaces and which can be extremely useful in the catalytic screening process by reducing the computational time for these type of surfaces. We find that, the band gap directly affects the binding energies of elector donor and acceptor fragments and we generalize the observations found for Lewis acid-base pairs. In the first part, we describe the linear correlation between the stabilization energy and the band gap of the oxides. In the second part, we explain the origin of this scaling relation in terms of shift of the Fermi level due to the adsorption of electron acceptors (A) and electron donor (D) fragments. In the last part, we report some 
considerations on the implication that this has on understanding bindings on semiconductors.

The relaxed structures and data presented here have been collected in a database which is available at the address http://nano.ku.dk/english/research/theoretical-electrocatalysis/ katladb/. ${ }^{39}$ Scripts to run the calculations and analyze the results have been also included, together with the input structures.

\section{Computational Methods}

We perform density functional theory (DFT) calculations using the GPAW code ${ }^{40,41}$ and the Atomic Simulation Environment (ASE). ${ }^{42}$ Each structure has been relaxed until the forces on all atoms were $<0.05 \mathrm{eV} / \AA$, using the revised Perdew-Burke-Ernzerhof (RPBE) exchange-correlation functional, ${ }^{43}$ a uniform real spaced grid with a spacing of $0.2 \AA$, and one k-point $(\Gamma)$, except for $\mathrm{RuO}_{2}$ for which a $4 \times 4 \times 1$ Monkhorst-Pack grid has been used. ${ }^{44}$ A dipole correction has also been included to eliminate the interaction among repeated slabs which are separated by at least $14 \AA$. Due to the well-known problem of the underestimation of the band gap, for a more accurate description of the electronic properties, like DOS and band gap, we perform hybrid functional calculations in the framework of the range-separated hybrid functional by Heyd, Scuseria, and Ernzerhof (HSE06). ${ }^{45,46}$ The wavefunctions were expanded in a plane-wave basis with a $500 \mathrm{eV}$ cutoff and we use one k-point $(\Gamma)$. The HSE06 calculations have been performed non-self-consistently from the PBE ground state density and wavefunctions. Even if the non-self-consistent HSE06 band gaps are rather accurate,${ }^{47,48}$ the total energies are not calculated in a correct way because they depend on both the position on the valence and conduction bands and on the electron density which is not updated self-consistently. To demonstrate that the scaling relationships between the interaction energy and band gap are similar despite the underestimation of the band gap typical of PBE calculations and considering that, at this stage, it is computationally too demanding to perform self-consistent HSE06 calculations, we apply the Hubbard correction 
to the results obtained using the Perdew-Burke-Ernzerhof functional (PBE) ${ }^{49}$ and fitting the value of $\mathrm{U}$ to obtain electronic properties (gap and DOS) similar to the ones calculated using HSE06. This is aimed to show that even computationally cheaper calculations (PBE and RPBE) give the same trends of more sophisticated results. More details are available in the Supplementary Information.

\section{Results and discussion}

Various band gap oxides (alkali rock-salts, like $\mathrm{MgO}, \mathrm{CaO}, \mathrm{SrO}, \mathrm{BaO}$, and $\mathrm{MgS}$, $\mathrm{TiO}_{2}$ in the rutile structure, the wurtzite $\mathrm{ZnO}$, the sesquioxide $\mathrm{La}_{2} \mathrm{O}_{3}$, perovskites such as $\mathrm{SrTiO}_{3}$, $\mathrm{BaHfO}_{3}$, and $\left.\mathrm{KTaO}_{3}\right)$ and different fragments $\left(\mathrm{H}^{*}, \mathrm{CH}_{3} \mathrm{C}^{*} \mathrm{O}, \mathrm{HO}^{*}, \mathrm{Cl}^{*}, \mathrm{CH}_{3} \mathrm{O}^{*}\right.$, in which the asterisk, $*$, indicates the active site of the fragment that binds on the oxide) have been investigated. We cut non-polar surfaces and we select different adsorption sites, on which the fragment exhibits properties of electron acceptor or donor. We consider different configurations of fragments, either alone in the cell or forming pairs or multiple adsorptions (a more detailed description has been reported in the Supporting Information).

There are two ways to calculate the dissociation energies for the two fragments: (i) each fragment is adsorbed alone on a slab and (ii) the fragments are coadsorbed. In the former case (i), the adsorption energy of the electron acceptor-donor (AD) pair is calculated as:

$$
\Delta E_{\mathrm{ads}}^{\mathrm{A}, \mathrm{D}}=E_{\mathrm{tot}}^{\mathrm{A}^{*}}+E_{\mathrm{tot}}^{\mathrm{D}^{*}}-2 E_{\mathrm{tot}}^{\mathrm{slab}}-E_{\mathrm{tot}}^{\mathrm{AD}},
$$

where $\Delta E_{\mathrm{ads}}^{\mathrm{A}, \mathrm{D}}$ is the adsorption energy of the single fragments $\mathrm{A}$ and $\mathrm{D}, E_{\mathrm{tot}}^{\mathrm{A}^{*}}$ and $E_{\mathrm{tot}}^{\mathrm{D}^{*}}$ is the DFT total energy of the slab with the fragments A and D adsorbed, $E_{\mathrm{tot}}^{\mathrm{slab}}$ is the energy of the clean slab, and $E_{\mathrm{tot}}^{\mathrm{AD}}$ is the energy of the undissociated $\mathrm{AD}$ molecule in the gas phase. In the latter, when the fragments are coadsorbed (ii), the dissociation energy is obtained as:

$$
\Delta E_{\mathrm{ads}}^{\mathrm{AD}}=E_{\mathrm{tot}}^{\mathrm{A}^{*} \mathrm{D}^{*}}-E_{\mathrm{tot}}^{\mathrm{slab}}-E_{\mathrm{tot}}^{\mathrm{AD}},
$$


where $\Delta E_{\mathrm{ads}}^{\mathrm{AD}}$ is the adsorption energy of the fragments $\mathrm{A}$ and $\mathrm{D}$ together and $E_{\mathrm{tot}}^{\mathrm{A}^{*} \mathrm{D}^{*}}$ is the total energy of the slab with the two fragments coadsorbed.

The difference between these two energies is thus:

$$
\begin{aligned}
E_{\text {diff }} & =\Delta E_{\mathrm{ads}}^{\mathrm{A}, \mathrm{D}}-\Delta E_{\mathrm{ads}}^{\mathrm{AD}}= \\
& =E_{\mathrm{tot}}^{\mathrm{A}^{*}}+E_{\mathrm{tot}}^{\mathrm{D}^{*}}-E_{\mathrm{tot}}^{\mathrm{A}^{*} \mathrm{D}^{*}}-E_{\mathrm{tot}}^{\mathrm{slab}} .
\end{aligned}
$$

A positive $E_{\text {diff }}$ indicates that the energy released by the dissociative adsorption is much lower than the sum of the energies of adsorbing the fragments on separate (but identical) surfaces. We are thus dealing with a cooperative adsorption between the fragments.

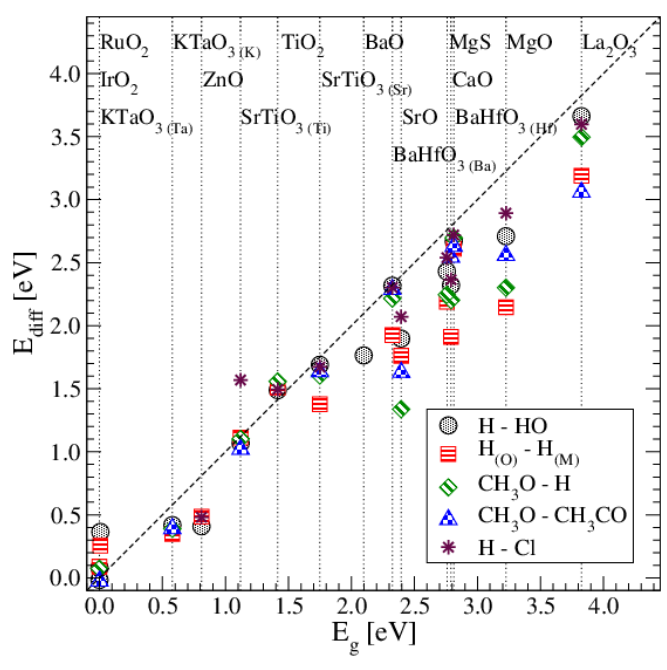

(a) PBE

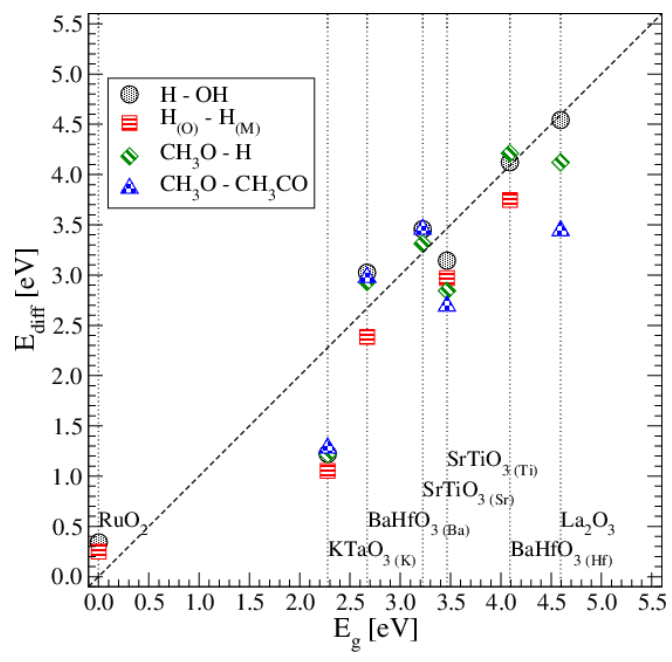

(b) $\mathrm{PBE}+\mathrm{U}$

Figure 1: Interaction energy, $E_{\text {diff }}$, as a function of the band gap, $E_{\mathrm{g}}$, of the clean surface for the studied set of semiconductors and insulators using PBE (a) and PBE $+\mathrm{U}(\mathrm{b})$ as exchangecorrelation functionals. The Hubbard correction has been applied only on slabs where the valence band is composed of oxygen $p$-states and the conduction band of metal $d$-states with a $\mathrm{U}$ value that well reproduce the band gap calculated with the hybrid exchange-correlation functional HSE06. For the perovskite slabs, the termination of the surface (A or B atom of the $\mathrm{ABO}_{3}$ unit cell) is reported in parentheses. When not indicated, $\mathrm{H}$ and $\mathrm{CH}_{3} \mathrm{CO}$ are adsorbed on top oxygen, while $\mathrm{Cl}, \mathrm{HO}$, and $\mathrm{CH}_{3} \mathrm{O}$ on top metal. When required, the adsorption site of $\mathrm{H}$ (oxygen or metal) is indicated in parentheses. The interaction energy correlates with the calculated band gap of the clean surface. The one-to-one relation between the interaction energy and the band gap is drawn with a dashed line.

The correlation between the interaction energy, $E_{\text {diff }}$, and the band gap is shown in 
Figure 1 using $\mathrm{PBE}$ and $\mathrm{PBE}+\mathrm{U}$ as exchange-correlation functional. We can distinguish two different behaviors: (i) conductors, like $\mathrm{IrO}_{2}$ and $\mathrm{RuO}_{2}$, for which the interaction energy is rather small (of the order of tenths of eV) and (ii) semiconductors, such as alkali oxides and perovskites, for which the interaction energy is of the order of eVs. The magnitude of the interaction energy is influenced by many factors, such as the element forming the oxide, the ionization potential or the electron affinity of the fragments, i.e. the ability to donate or accept a charge (strength of the fragment). The amount of charge transfer is also influenced by a structural relaxations of the oxide (polaronic distortions), ${ }^{38}$ the nature of the oxide (reducible or nonreducible), the surface exposed, and the presence of defects. ${ }^{50}$ Despite of these, we can identify a one-to-one scaling between the interaction energy and the band gap (the discrepancies from the one-to-one scaling are caused by the effects mentioned above). We have noticed that the relaxation has the effect of shifting the states in the band gap closer to the band edges of the clean surface. This would explain why the band gap of the clean metal slab is a good descriptor for the interaction energy despite the effects mentioned above.

To explain this behavior, we have to consider what happens at the valence and conduction band states and at the position of the Fermi level before and after the coadsorption (a sketch is shown in Figure 2 and the densities of states (DOS) of $\mathrm{MgO}$ with different adsorbates in Figure 3. More example of DOS are shown in the Supporting Information). When an electron acceptor or donor fragment is adsorbed on an intrinsic semiconductor, an electron transfers from the slab to the fragment or vice versa with the consequence that the Fermi level down-(up-) shifts to the valence (conduction) band edge. When an electron acceptordonor pair is adsorbed an electron is transferred from the electron donor to the electron acceptor fragment through the surface. Since no electrons have been added to (removed from) the surface, the Fermi level remains unchanged. The different behavior regarding the shift of the Fermi level in the two cases is responsible for the cooperative effect. In fact, when the two fragments are at infinite distance (Eq. 1), the electron transfer shifts the Fermi 

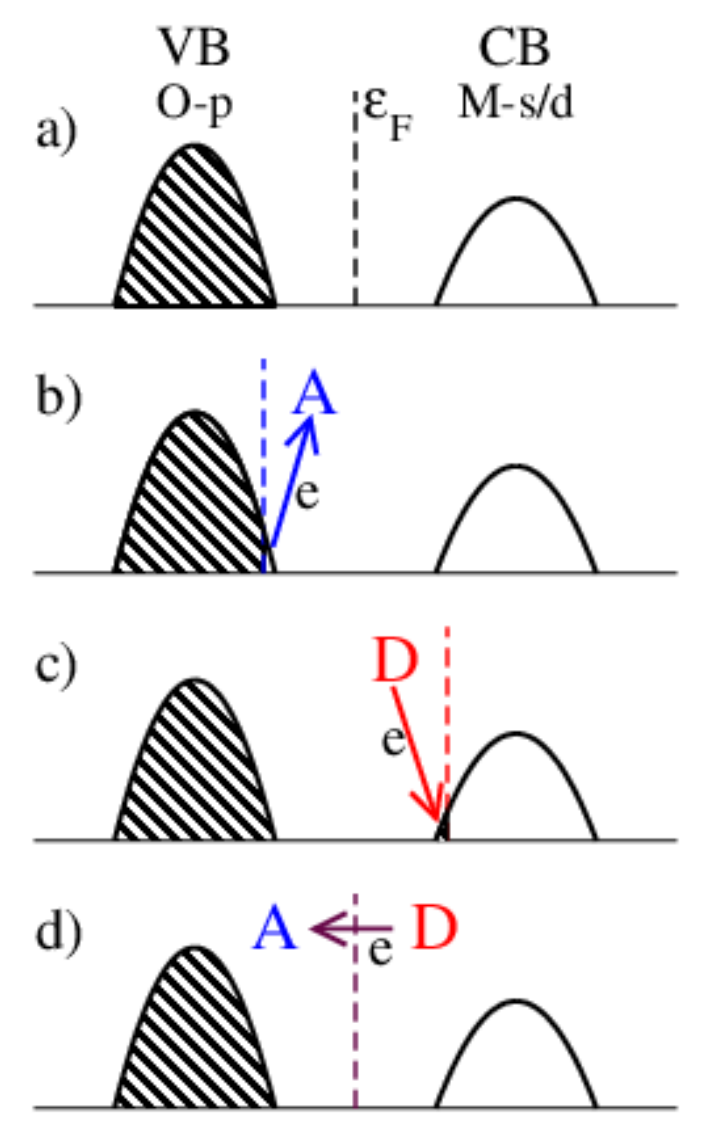

Figure 2: Sketch of the valence and conduction band states for different surfaces. (a) Intrinsic semiconductor: the Fermi level is in the middle of the band gap between the valence (VB) and the conduction bands (CB). (b) An electron acceptor fragment (A) is adsorbed: an electron moves from the VB to the fragment and the Fermi level of the slab down-shifts. (c) An electron donor (D) is adsorbed: an electron transfers to the CB of the slab from the fragment and the slab Fermi level up-shift. (d) Coadsorption of an AD pair: an electron transfers from the electron donor to the electron acceptor fragments and the shift in the position of the Fermi level is basically zero. 
level, while when the two fragments are at finite but still not-interacting distance (Eq. 2), the Fermi level does not move.

The densities of states (DOS) of the considered fragments and their combinations on $\mathrm{MgO}$ are shown in Figure 3. The DOS of the clean surface is plotted in black, the Fermi level (dashed line) is in the middle of the band gap because we are dealing with an intrinsic semiconductor. Now, one hydrogen is adsorbed. Since its electronegativity is smaller than the one of the oxygen on top which it is adsorbed $(1 \mathrm{H}(1 \mathrm{O})$, in figure), hydrogen behaves as an electron donor and one electron transfers from hydrogen to oxygen. This changes the oxidation state of the nearby atoms. The extra electron ends up in the conduction band and the Fermi level up-shifts to the conduction band. We found similar effects for other electron donor fragments like $\mathrm{CH}_{3} \mathrm{CO}$ or when more electron donor fragments are coadsorbed, like two hydrogen atoms on top oxygens $(2 \mathrm{H}(2 \mathrm{O}))$. When an electron acceptor fragment, like HO or $\mathrm{CH}_{3} \mathrm{O}$, is adsorbed, an electron transfers from the semiconductor to the adsorbate and the Fermi level down-shifts to the valence band edge. Hydrogen can also behave as an electron acceptor when adsorbed on top metal, $1 \mathrm{H}(1 \mathrm{Mg})$, which has a smaller electronegativity. Now the extra electron ends up in the valence band and the Fermi level down-shifts. If an electron acceptor and an electron donor are coadsorbed, as for example 1H,1HO in the figure, an electron transfers from the electron donor $(\mathrm{H})$ to the electron acceptor fragment $(\mathrm{HO})$. In these cases, there is no shift of the Fermi level.

To predict the shift of the Fermi level when more than two fragments are coadsorbed, we have to count how many fragments behave as electron donor and how many as electron acceptors. If the number of donor is larger than the number of acceptors, the Fermi level up-shifts to the conduction band, vice versa when there are more acceptors than donor, the Fermi level down-shifts to the valence band. The Fermi level does not shift when the number of acceptors is equal to the number of donors.

We also consider processes with more than one electron transferred, like, for example, when 4 hydrogens are adsorbed, 2 on top metal and 2 on top oxygen (two electrons transfer). 


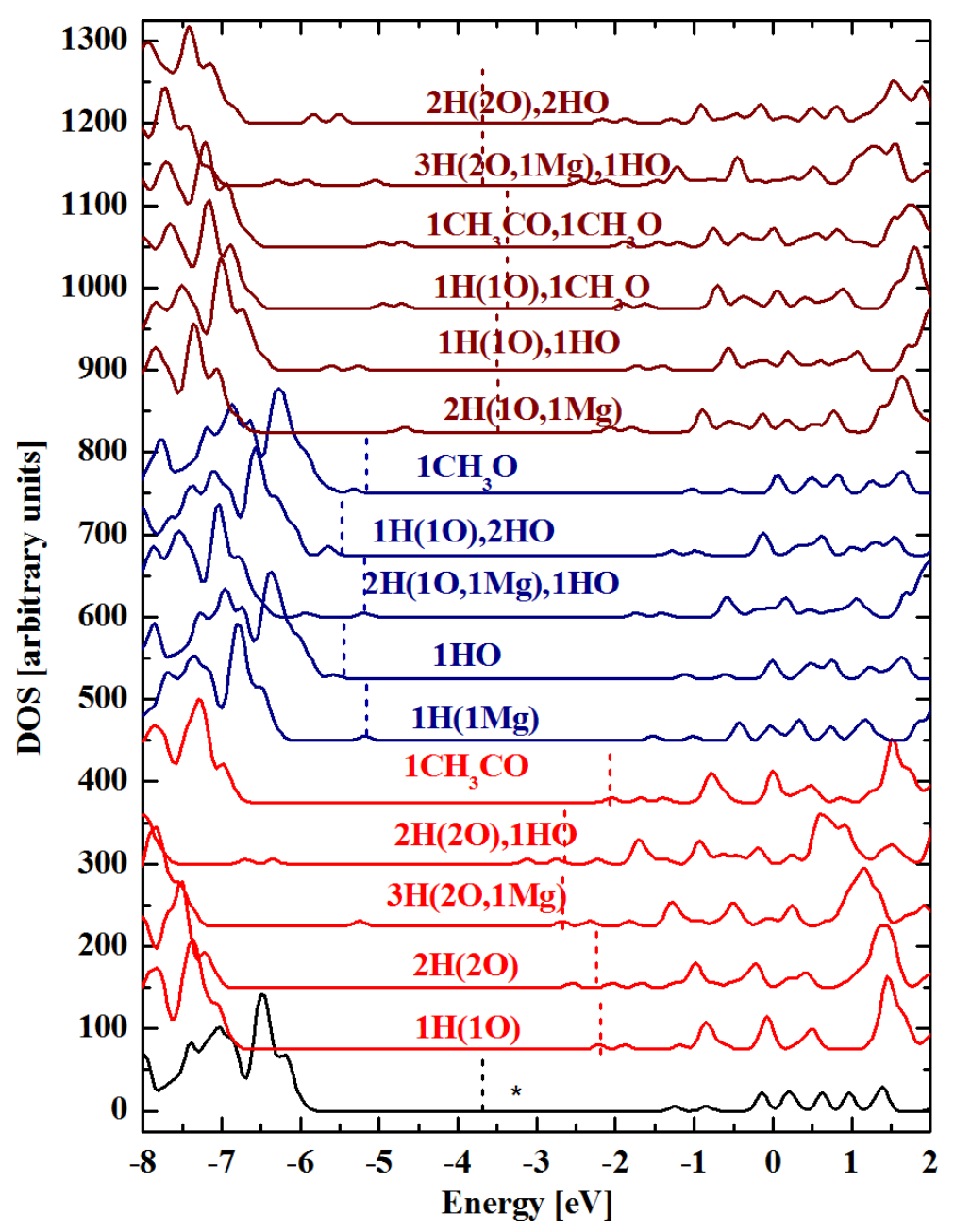

Figure 3: Density of states for various combinations of fragments on $\mathrm{MgO}$ calculated using HSE06. In parenthesis is indicated the adsorption site when the fragment is not adsorbed on its most stable adsorption site as indicated in the Methods section (for example, $2 \mathrm{H}(1 \mathrm{O}, 1 \mathrm{Mg}), 1 \mathrm{HO}$ means that two hydrogens, one on top oxygen and one on top metal and one HO group on top metal are adsorbed). The clean slab is labeled with a $*$. The Fermi levels are indicated by dashed lines, and the color of the DOS depends on the shift of the Fermi level with respect to the clean slab: in red, when the charges donated by the fragments are larger then the ones accepted by them so that Fermi level shifts up, in blue, the opposite, when the charges donated by the fragments are smaller then the ones accepted by them so that Fermi level shifts down, and in brown, then the charges donated and accepted by the fragments are balanced. The zero on the x-axis correspond to the vacuum level. The top view of the systems considered here is shown in Figure S2. 


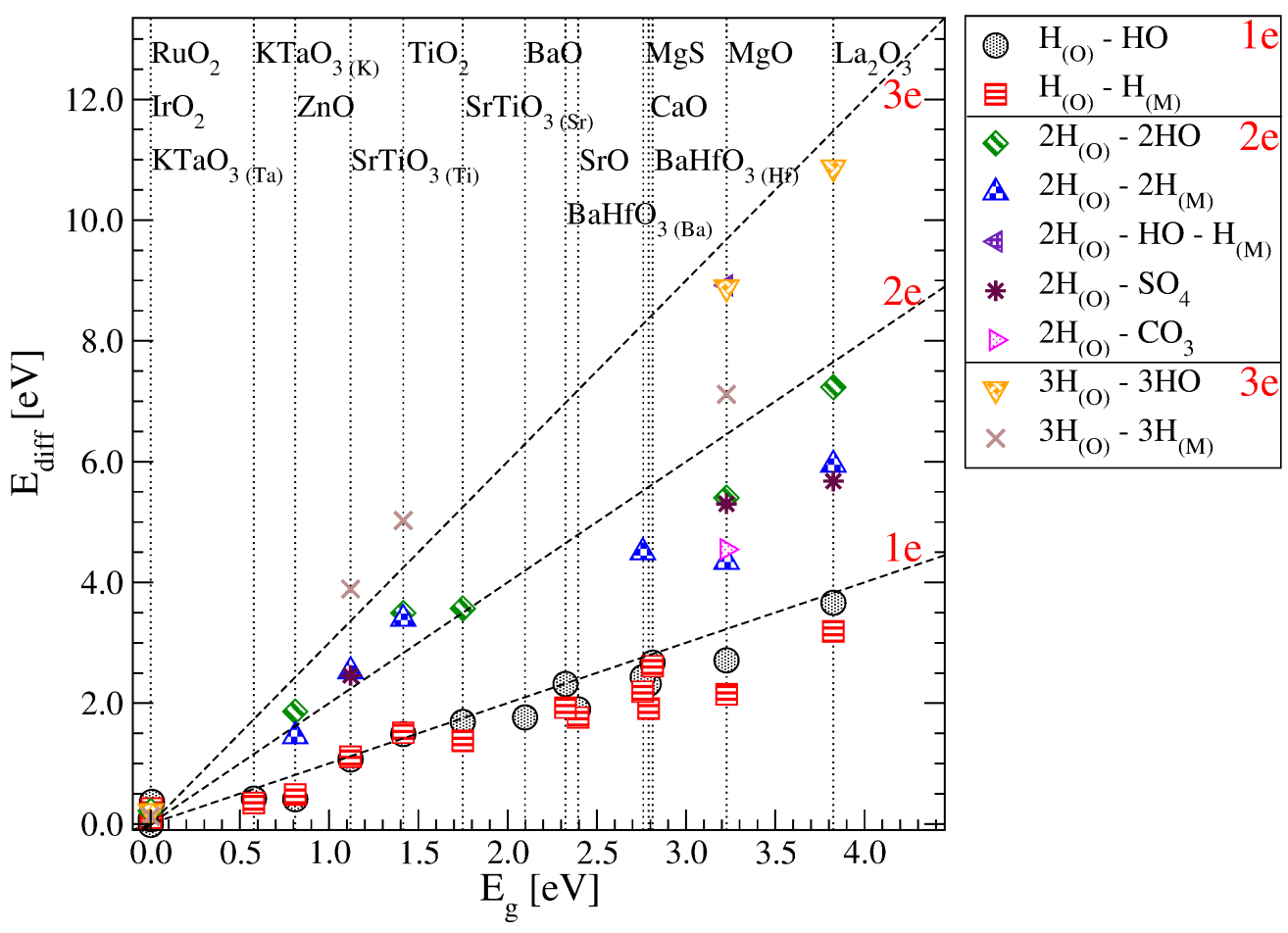

Figure 4: Interaction energy at the PBE level as a function of the band gap of the clean surface for multiple electron transfer processes. $E_{\text {diff }}$ scales with the band gap multiplied by the number of electron transfer (dashed lines).

Figure 4 shows the correlation between the interaction energy and the band gap for the two electron transfer process. Since two electrons are transferred, the interaction energy scales with twice the size of the band gap as the stabilization equals the size of the band gap for each fragment pair. The same happens when three electrons are transferred. We can thus generalize that the interaction energy scales with the band gap multiplied by the number of electrons which transfer between the electron donor and electron acceptor fragment(s).

In some cases (Figure 1), the interaction energy deviates from the band gap with the increase of the size of the gap. This can be caused by different factors: (i) there are states originated by the fragments inside the band gap (see the DOS in Figure 3) which reduce the gap of few tenths of $\mathrm{eV}$ so that the energy level where the electron transfers do no longer correspond to the valence or conduction band of the clean slab but to the extra states created by the adsorbate and (ii) the fragments have different strength, due to different electronegativities of the chemical elements involved, so that, in some cases, only a fraction 
of an electron is transferred.

We note that GGA exchange-correlation functionals underestimate the magnitude of the band gap, however the correlation between the band gap and the cooperative interaction is still valid no-matter the underestimation. In fact, when the gap is underestimated, $E_{\text {diff }}$ is underestimated accordingly.

\section{Implications for Design of Novel Catalysts}

The majority of the thermodynamic scaling relations in heterogeneous catalysis were developed for pure transition metal surfaces and implied scalings between adsorption energies of diverse fragments, e.g. binding energies of molecular fragments and the central atom, ${ }^{51}$ across a series of flat and stepped transition metal surfaces or between oxygen and oxygenates on the same transition metal surfaces. ${ }^{17}$ This concepts have then be extended to other catalytic surfaces, like transition metal oxides, nitrides, and so on. ${ }^{9,15,16}$ Modelling coadsorbed fragments on metals is made simple by the fact that adsorption and dissociation energies are calculated as sum of energies of isolated fragments which gives the same results as energies calculated with both the species in the same cell, but at a significantly reduced computational cost.

We have shown here that semiconductors behave in a more complex way and we thus need to go beyond the study of surfaces with single adsorbates towards the prediction of thermodynamic steps that involve interactions between donor and acceptor fragments, which requires larger unit cells and higher computational cost. We can reduce the complexity of reactions on semiconductors by understanding that the adsorption of acceptor-donor pairs, $\Delta E_{\mathrm{ads}}^{\mathrm{AD}}$, is energetically highly favored compared to unpaired adsorbates, $E_{\text {tot }}^{\mathrm{A}^{*}}$ and $E_{\text {tot }}^{\mathrm{D}^{*}}$ and this cooperative energy corresponds to the value of the band gap, $E_{\mathrm{g}}$ :

$$
\Delta E_{\mathrm{ads}}^{\mathrm{AD}}=E_{\mathrm{tot}}^{\mathrm{A}^{*}}+E_{\mathrm{tot}}^{\mathrm{D}^{*}}+E_{\mathrm{g}} .
$$


The complexity is increased by the fact that some fragments can behave both as acceptors and as donors, like hydrogen, and the fact that the metal forming the oxide can be reduced. On $\mathrm{MgO}$ or $\mathrm{TiO}_{2}$, for example, a single hydrogen adsorbs on top oxygen. When two hydrogens act like donors, i.e. are coadsorbed on top oxygen, the Fermi level stays at the valence band, while when an acceptor-donor pair is adsorbed, i.e. one hydrogen on top oxygen and one on top metal, the Fermi level is found in the middle of the gap. As shown here, it is energetically more favorable to adsorb an hydrogen acceptor-donor pair on $\mathrm{MgO} .{ }^{52-55}$ Similar behavior has been found on $\mathrm{MgO}(100)$ and on $\mathrm{La}_{2} \mathrm{O}_{3}$ for methane dissociation and $\mathrm{CeO}_{2}$ for halogen dissociation. ${ }^{28,37,56}$ On $\mathrm{TiO}_{2}$, instead, the most stable configuration has both hydrogens adsorbed on oxygen as electron donor. This has been explained by the fact that Ti reduces. ${ }^{57,58}$

\section{Conclusions}

The investigation of descriptors and scaling relationships is extremely useful to understand catalysis and speed-up the search for novel and better catalysts.

In this paper, we have shown that the band gap is a descriptor for cooperative energy which is the difference between the binding energies calculated for an electron donor-acceptor pair on band gap oxides at infinite and at finite but non-interacting distances. To be more precise, the interaction energy is proportional to the band gap times the number of electron transferred. For conductive oxides, as well as for semiconducting surfaces with a preadsorbed fragment, the interaction energy tends to zero.

This has been explained considering the shift of the Fermi level. When a single fragment is adsorbed, the Fermi level shifts to the edge of the conduction or valence bands for an electron donor or acceptor fragment, respectively. If a pair is coadsorbed, the shift of the Fermi level is almost null and the Fermi level stays in the middle of the gap. It is thus important to consider which of the fragment is a donor and which one is an acceptor to 
model correctly these oxide systems.

These findings are important for understanding the binding on semiconducting surfaces and they have practical relevance and implications on how to simplify and understand chemical reactions on semiconductors. The most likely reactions, in fact, conserve an equal amount of acceptors and donors. By using the band gap as a descriptor for the interaction energy, we can also estimate the energetics without having to run expensive and time consuming calculations.

\section{Supporting Information Available}

The Supporting Information available free of charge.

The following figures are included.

- Figure S1: Interaction energy as a function of the band gap calculated with PBE and RPBE as exchange-correlation functionals.

- Figure S2: Top-view of the position of the fragments on $\mathrm{MgO}(100)$. All the structures investigated in this manuscript are also available online at http://nano.ku.dk/ english/research/theoretical-electrocatalysis/katladb/.

- Figure S3: Top-view of the position of the fragments on $\mathrm{TiO}_{2}(110)$.

- Figure S4: Projected densities of states for $\mathrm{MgO}$ and some significant fragments.

- Figure S5: DOS and PDOS of $\mathrm{TiO}_{2}$.

- Figure S6: DOS and PDOS of $\mathrm{SrTiO}_{3}$ terminated with Ti and Sr.

- Figure S7: DOS and PDOS of $\mathrm{La}_{2} \mathrm{O}_{3}$.

This material is available free of charge via the Internet at http://pubs.acs.org/. 


\section{Acknowledgement}

The authors thank Karsten W. Jacobsen for the useful discussions. I.E.C. and J.R. acknowledge support from BMW Research and Carlsberg Foundation (grant CF15-0165). I.C.M. and V.P. acknowledge support from the Romanian National Authority for Scientific Research, CNCS-UEFISCDI under project number PN-II-RU-PD-2012-28/26.04.2013.

\section{References}

(1) Hammer, B.; Nørskov, J. Impact of Surface Science on Catalysis; Advances in Catalysis; Academic Press, 2000; Vol. 45; pp $71-129$.

(2) Greeley, J.; Mavrikakis, M. Alloy Catalysts Designed from First Principles. Nat Mater 2004, 3, 810-815.

(3) Neurock, M. Perspectives on the First Principles Elucidation and the Design of Active Sites. J Catal 2003, 216, $73-88$.

(4) Nørskov, J. K.; Bligaard, T.; Rossmeisl, J.; Christensen, C. H. Towards the Computational Design of Solid Catalysts. Nat Chem 2009, 1, 37-46.

(5) Guo, Z.; Liu, B.; Zhang, Q.; Deng, W.; Wang, Y.; Yang, Y. Recent Advances in Heterogeneous Selective Oxidation Catalysis for Sustainable Chemistry. Chem. Soc. Rev. 2014, 43, 3480-3524.

(6) Calle-Vallejo, F.; Koper, M. T. First-principles Computational Electrochemistry: Achievements and Challenges. Electrochim Acta 2012, 84, 3-11.

(7) Curtarolo, S.; Hart, G. L. W.; Nardelli, M. B.; Mingo, N.; Sanvito, S.; Levy, O. The High-throughput Highway to Computational Materials Design. Nat Mater 2013, 12, $191-201$. 
(8) Studt, F.; Abild-Pedersen, F.; Bligaard, T.; Sørensen, R. Z.; Christensen, C. H.; Nørskov, J. K. Identification of Non-Precious Metal Alloy Catalysts for Selective Hydrogenation of Acetylene. Science 2008, 320, 1320-1322.

(9) Greeley, J. Theoretical Heterogeneous Catalysis: Scaling Relationships and Computational Catalyst Design. Annu Rev Chem Biomol 2016, 7, 605-635.

(10) Hansgen, D. A.; Vlachos, D. G.; Chen, J. G. G. Using First Principles to Predict Bimetallic Catalysts for the Ammonia Decomposition Reaction. Nat Chem 2010, 2, 484-489.

(11) Guo, W.; Vlachos, D. G. Patched Bimetallic Surfaces Are Active Catalysts for Ammonia Decomposition. Nat Comm 2015, 6 .

(12) Besenbacher, F.; Chorkendorff, I.; Clausen, B.; Hammer, B.; Molenbrock, A.; Nørskov, J.; Stensgaard, I. Design of a Surface Alloy Catalyst for Steam Reforming. Science 1998, 279, 1913-1915.

(13) Zaffran, J.; Michel, C.; Auneau, F.; Delbecq, F.; Sautet, P. Linear Energy Relations As Predictive Tools for Polyalcohol Catalytic Reactivity. ACS Catal 2014, 4, 464-468.

(14) Lopez, N.; Almora-Barrios, N.; Carchini, G.; Blonski, P.; Bellarosa, L.; GarciaMuelas, R.; Novell-Leruth, G.; Garcia-Mota, M. State-of-the-art and Challenges in Theoretical Simulations of Heterogeneous Catalysis at the Microscopic Level. Catal. Sci. Technol. 2012, 2, 2405-2417.

(15) Fernández, E. M.; Moses, P. G.; Toftelund, A.; Hansen, H. A.; Martínez, J. I.; AbildPedersen, F.; Kleis, J.; Hinnemann, B.; Rossmeisl, J.; Bligaard, T. et al. Scaling Relationships for Adsorption Energies on Transition Metal Oxide, Sulfide, and Nitride Surfaces. Angew Chem Int Edit 2008, 120, 4761-4764. 
(16) Man, I. C.; Su, H.-Y.; Calle-Vallejo, F.; Hansen, H. A.; Martinez, J. I.; Inoglu, N. G.; Kitchin, J.; Jaramillo, T. F.; Nørskov, J. K.; Rossmeisl, J. Universality in Oxygen Evolution Electrocatalysis on Oxide Surfaces. ChemCatChem 2011, 3, 1159-1165.

(17) Calle-Vallejo, F.; Loffreda, D.; Koper, M. T. M.; Sautet, P. Introducing Structural Sensitivity Into Adsorption-energy Scaling Relations by Means of Coordination Numbers. Nat Chem 2015, 7, 403-410.

(18) Zaffran, J.; Michel, C.; Delbecq, F.; Sautet, P. Trade-Off between Accuracy and Universality in Linear Energy Relations for Alcohol Dehydrogenation on Transition Metals. $J$ Phys Chem C 2015, 119, 12988-12998.

(19) Nørskov, J.; Bligaard, T.; Logadottir, A.; Bahn, S.; Hansen, L.; Bollinger, M.; Bengaard, H.; Hammer, B.; Sljivancanin, Z.; Mavrikakis, M. et al. Universality in Heterogeneous Catalysis. J Catal 2002, 209, 275 - 278.

(20) Jones, G.; Studt, F.; Abild-Pedersen, F.; Nørskov, J. K.; Bligaard, T. Scaling Relationships for Adsorption Energies of $\mathrm{C}_{2}$ Hydrocarbons on Transition Metal Surfaces. Chem Eng Sci 2011, 66, $6318-6323$.

(21) Greeley, J.; Jaramillo, T. F.; Bonde, J.; Chorkendorff, I.; Nørskov, J. K. Computational High-throughput Screening of Electrocatalytic Materials for Hydrogen Evolution. Nat Mater 2006, 5, 909-913.

(22) Calle-Vallejo, F.; Martínez, J. I.; García-Lastra, J. M.; Rossmeisl, J.; Koper, M. T. M. Physical and Chemical Nature of the Scaling Relations between Adsorption Energies of Atoms on Metal Surfaces. Phys. Rev. Lett. 2012, 108, 116103.

(23) Studt, F.; Sharafutdinov, I.; Abild-Pedersen, F.; Elkjær, C. F.; Hummelshøj, J. S.; Dahl, S.; Chorkendorff, I.; Nørskov, J. K. Discovery of a Ni-Ga catalyst for Carbon Dioxide Reduction to Methanol. Nat Chem 6, 320-324. 
(24) Rossmeisl, J.; Logadottir, A.; Nørskov, J. Electrolysis of Water on (Oxidized) Metal Surfaces. Chem Phys 2005, 319, 178 - 184.

(25) Broqvist, P.; Panas, I.; Fridell, E.; Persson, H. NOxStorage on BaO(100) Surface from First Principles: a Two Channel Scenario. J Phys Chem B 2002, 106, 137-145.

(26) Ménétrey, M.; Markovits, A.; Minot, C. Adsorption of Chlorine and Oxygen Atoms on Clean and Defective Rutile-TiO2 (110) and MgO (100) Surfaces. J Mol Struc-Theochem 2007, 808, $71-79$.

(27) Metiu, H.; Chrétien, S.; Hu, Z.; Li, B.; Sun, X. Chemistry of Lewis Acid-Base Pairs on Oxide Surfaces. J Phys Chem C 2012, 116, 10439-10450.

(28) Hu, Z.; Metiu, H. Halogen Adsorption on $\mathrm{CeO}_{2}$ : The Role of Lewis Acid-Base Pairing. J Phys Chem C 2012, 116, 6664-6671.

(29) Li, B.; Metiu, H. Does Halogen Adsorption Activate the Oxygen Atom on an Oxide Surface? I. A Study of $\mathrm{Br}_{2}$ and $\mathrm{HBr}$ Adsorption on $\mathrm{La}_{2} \mathrm{O}_{3}$ and $\mathrm{La}_{2} \mathrm{O}_{3}$ Doped with $\mathrm{Mg}$ or Zr. J Phys Chem C 2012, 116, 4137-4148.

(30) Honkala, K.; Hellman, A.; Grönbeck, H. Water Dissociation on MgO/Ag(100): Support Induced Stabilization or Electron Pairing? J Phys Chem C 2010, 114, 7070-7075.

(31) Schneider, W. F.; Hass, K. C.; Miletic, M.; Gland, J. L. Dramatic Cooperative Effects in Adsorption of NOxon $\mathrm{MgO}(001)$. J Phys Chem B 2002, 106, 7405-7413.

(32) Miletic, M.; Gland, J. L.; Hass, K. C.; Schneider, W. F. First-Principles Characterization of NOx Adsorption on MgO. J Phys Chem B 2003, 107, 157-163.

(33) Schneider, W. F. Qualitative Differences in the Adsorption Chemistry of Acidic $\left(\mathrm{CO}_{2}\right.$, $\left.\mathrm{SO}_{x}\right)$ and Amphiphilic $\left(\mathrm{NO}_{x}\right)$ Species on the Alkaline Earth Oxides. J Phys Chem B 2004, 108, 273-282. 
(34) Broqvist, P.; Panas, I.; Grönbeck, H. The Nature of $\mathrm{NO}_{x}$ Species on $\mathrm{BaO}(100)$ : An Ab Initio Molecular Dynamics Study. J Phys Chem B 2005, 109, 15410-15416.

(35) Grönbeck, H.; Broqvist, P.; Panas, I. Fundamental aspects of NOx adsorption on BaO. Surf Sci 2006, 600, 403-408.

(36) Abrahamsson, B.; Grönbeck, H. NOxAdsorption on ATiO3(001) Perovskite Surfaces. J Phys Chem C 2015, 119, 18495-18503.

(37) Chrétien, S.; Metiu, H. Acid-Base Interaction and Its Role in Alkane Dissociative Chemisorption on Oxide Surfaces. J Phys Chem C 2014, 118, 27336-27342.

(38) den Bossche, M. V.; Grönbeck, H. Adsorbate Pairing on Oxide Surfaces: Influence on Reactivity and Dependence on Oxide, Adsorbate Pair, and Density Functional. J Phys Chem C 2017, 121, 8390-8398.

(39) Online Supplementary Information, KatlaDB - Theoretical Catalysis Database. http://nano.ku.dk/english/research/theoretical-electrocatalysis/katladb, Nano-Science Center, Department of Chemistry, University of Copenhagen, accessed June 2017.

(40) Mortensen, J. J.; Hansen, L. B.; Jacobsen, K. W. Real-space Grid Implementation of the Projector Augmented Wave Method. Phys. Rev. B 2005, 71, 035109.

(41) Enkovaara, J.; Rostgaard, C.; Mortensen, J. J.; Chen, J.; Dułak, M.; Ferrighi, L.; Gavnholt, J.; Glinsvad, C.; Haikola, V.; Hansen, H. A. et al. Electronic Structure Calculations with GPAW: a Real-space Implementation of the Projector Augmentedwave Method. J Phys: Condens Mat 2010, 22, 253202.

(42) Larsen, A. H.; Mortensen, J. J.; Blomqvist, J.; Castelli, I. E.; Christensen, R.; Dulak, M.; Friis, J.; Groves, M. N.; Hammer, B.; Hargus, C. et al. The Atomic Simulation 
Environment - a Python Library for Working with Atoms. J Phys: Condens Mat 2017, 29, 273002.

(43) Hammer, B.; Hansen, L. B.; Nørskov, J. K. Improved Adsorption Energetics Within Density-functional Theory Using Revised Perdew-Burke-Ernzerhof Functionals. Phys. Rev. B 1999, 59, 7413-7421.

(44) Monkhorst, H. J.; Pack, J. D. Special Points for Brillouin-zone Integrations. Phys. Rev. B 1976, 13, 5188-5192.

(45) Heyd, J.; Scuseria, G. E.; Ernzerhof, M. Hybrid Functionals Based on a Screened Coulomb Potential. J. Chem. Phys. 2003, 118, 8207.

(46) Krukau, A. V.; Vydrov, O. A.; Izmaylov, A. F.; Scuseria, G. E. Influence of the Exchange Screening Parameter on the performance of Screened Hybrid Functionals. J. Chem. Phys. 2006, 125, 224106.

(47) Paier, J.; Marsman, M.; Hummer, K.; Kresse, G.; Gerber, I. C.; Ángyán, J. G. Screened Hybrid Density Functionals Applied to Solids. J Chem Phys 2006, 124, 154709.

(48) Castelli, I. E.; Hüser, F.; Pandey, M.; Li, H.; Thygesen, K. S.; Seger, B.; Jain, A.; Persson, K. A.; Ceder, G.; Jacobsen, K. W. New Light-Harvesting Materials Using Accurate and Efficient Bandgap Calculations. Adv Energy Mater 2015, 5, 1400915.

(49) Perdew, J. P.; Burke, K.; Ernzerhof, M. Generalized Gradient Approximation Made Simple. Phys. Rev. Lett. 1996, 77, 3865-3868.

(50) Pacchioni, G. Electronic Interactions and Charge Transfers of Metal Atoms and Clusters on Oxide Surfaces. Phys Chem Chem Phys 2013, 15, 1737.

(51) Abild-Pedersen, F.; Greeley, J.; Studt, F.; Rossmeisl, J.; Munter, T. R.; Moses, P. G.; Skúlason, E.; Bligaard, T.; Nørskov, J. K. Scaling Properties of Adsorption Energies for 
Hydrogen-Containing Molecules on Transition-Metal Surfaces. Phys. Rev. Lett. 2007, 99, 016105.

(52) Coluccia, S.; Boccuzzi, F.; Ghiotti, G.; Mirra, C. Evidence for Heterolytic Dissociation of $\mathrm{H}_{2}$ on the Surface of Thermally Activated MgO Powders. Z Phys Chem 1980, 121, $141-143$.

(53) Ito, T.; Kuramoto, M.; Yoshioka, M.; Tokuda, T. Active Sites for Hydrogen Adsorption on Magnesium Oxide. J Phys Chem 1983, 87, 4411-4416.

(54) Shluger, A. L.; Gale, J. D.; Catlow, C. R. A. Molecular Properties of the Magnesia Surface. J Phys Chem 1992, 96, 10389-10397.

(55) Gribov, E. N.; Bertarione, S.; Scarano, D.; Lamberti, C.; Spoto, G.; Zecchina, A. Vibrational and Thermodynamic Properties of $\mathrm{H}_{2}$ Adsorbed on $\mathrm{MgO}$ in the 300-20 K Interval. J Phys Chem B 2004, 108, 16174-16186.

(56) Anchell, J. L.; Morokuma, K.; Hess, A. C. An Electronic Structure Study of $\mathrm{H}_{2}$ and $\mathrm{CH}_{4}$ Interactions with $\mathrm{MgO}$ and Li-doped MgO Clusters. J Phys Chem 1993, 99, 6004-6013.

(57) Calatayud, M.; Markovits, A.; Menetrey, M.; Mguig, B.; Minot, C. Adsorption on Perfect and Reduced Surfaces of Metal Oxides. Catal Today 2003, 85, 125 - 143, Metallic Oxides: Filling the Gap between Catalysis and Surface Science.

(58) Leconte, J.; Markovits, A.; Skalli, M.; Minot, C.; Belmajdoub, A. Periodic Ab-initio Study of the Hydrogenated Rutile TiO2(110) Surface. Surf Sci 2002, 497, 194 - 204. 


\section{${ }_{401}$ TOC Graphic}

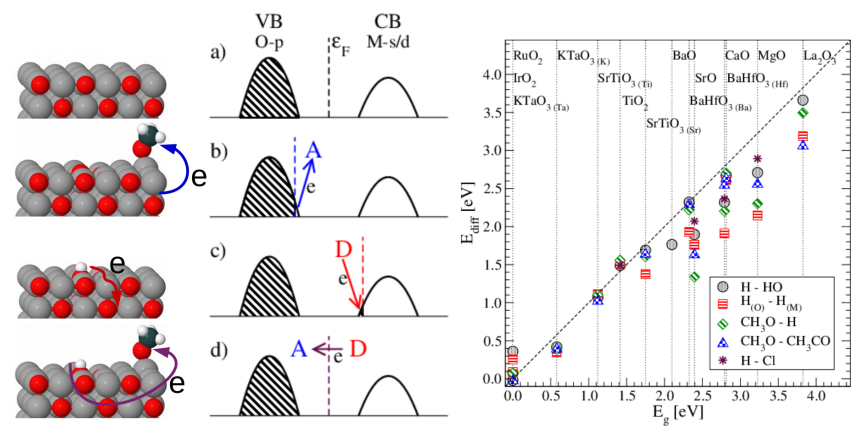

